\title{
Glaucoma Screening from a Public Health Clinic*
}

\author{
E. S. PERKINS, $†$ M.D., PH.D., F.R.C.S.
}

Brit. med. F., 1965, 1, 417-419

According to statistics published in 1956 (Sorsby, 1956), glaucoma is responsible for $13.6 \%$ of registrations of blindness. The same statistics showed that $40 \%$ of those blind from glaucoma had had no treatment, indicating that they were unaware of the seriousness of their eye trouble until they became blind.

Chronic glaucoma has an insidious onset, and the tragedy of the disease is that symptoms may not be noticed by the patient until severe damage to the optic nerve has taken place. Treatment at this stage can at best only halt the progression of the disease, and if blindness or serious visual defect is to be prevented the cases must be detected early. This can be achieved only by screening apparently normal populations.

At the instigation of Dr. C. Sharp, the Medical Officer of Health for Bedford, and with support from the Ministry of Health, a screening programme has been started in Bedford. Before going into details the aims of this particular survey should be made clear. They are to assess the incidence of glaucoma in a sample of the population over the age of 40 , to assess the available diagnostic methods for the detection of glaucoma, to discover practical ways in which glaucoma investigations can be organized within the structure of a local public health service, and, finally, to provide clinical material for research into the early stages in the development of the disease. It should be emphasized that the aim is not to find the largest number of cases of glaucoma in the shortest possible time. Mass-screening programmes are no doubt valuable in some circumstances, but the follow-up of doubtful cases is very difficult and the determination of the true incidence of glaucoma from such surveys is not easy.

Bedford is a fairly typical county town with a population of some 60,000. Apart from a recent influx of migrants from Europe, the West Indies, and Pakistan, the population is fairly static, so that long-term follow-up of cases should be possible. Public relations with the local health services are excellent and the people have to some extent been conditioned to accept surveys by the very successful diabetic survey recently done in Bedford.

\section{Glaucoma Survey}

The survey is held in one of the local authority health centres situated fairly centrally in the town. It is a modern building with excellent facilities. The survey staff consists of an ophthalmologist, a glaucoma technician, a nurse, and a secretary-receptionist.

After a publicity campaign in the local press and meetings with the general practitioners, opticians, and other interested groups, cards requesting an appointment for the tests were distributed to the general public. The response to the appeal for volunteers was excellent, and we soon had a waiting-list of some three to four weeks. Very little further publicity has been required to keep up the supply of volunteers, most of whom are now coming on the recommendation of people who have already been tested.

\footnotetext{
* Paper read in a symposium on glaucoma at the Annual Meeting of the British Medical Association, Manchester, 1964.

† Professor of Experimental Ophthalmology, Institute of Ophthalmology, University of London.
}

The most important evidence of glaucoma is a raised intraocular pressure. Numerous instruments have been designed for measuring this clinically: some of these are relatively simple, others very complex. For the purpose of this survey we chose to use the Goldmann applanation tonometer. Although this is expensive and its use requires considerable experience, it is undoubtedly the most accurate clinical instrument available and causes no discomfort to the patient.

On his appearance at the clinic the patient's name, address, and age and the address of his general practitioner are entered on a survey card by the secretary. She notes whether the patient has had glaucoma or has a family history of glaucoma. In the case of women a note is also made of the time of the last menstrual period.

The patient has Novesine drops put in each eye, and then fluorescein, in preparation for applanation tonometry, which is done by the technician. The patient is then seen by the ophthalmologist, who notes the tension readings recorded by the technician and does a rapid external examination and ophthalmoscopy. He also inquires whether the patient has had any attacks of blurred vision or has seen haloes. Any abnormal findings are noted, and if none are found the patient is discharged with a note informing him that there is no evidence of glaucoma, but also emphasizing that he has not had a complete ophthalmic examination. Patients with ocular abnormalities other than glaucoma which require treatment-for example, hypertensive retinopathy-are referred back to their doctor with a report.

Patients in whom glaucoma is suspected are given an appointment for the assessment clinic, the criteria for assessment being : (1) applanation reading of $21 \mathrm{~mm}$. Hg or above, (2) difference of $5 \mathrm{~mm}$. $\mathrm{Hg}$ between the two eyes, (3) suspicious disks, (4) history of haloes or attacks of blurred vision, and (5) family history of glaucoma.

Initially we had planned three screening clinics a week, one tonography clinic, and one assessment clinic. However, we found that using these criteria it was necessary to have at least two assessment clinics a week, and the screening rate is limited not so much by the number it is possible to screen but by the number it is possible to assess adequately. We find that the screening of 120 to 150 patients a week produces about 10 for assessment.

At the assessment clinic the patient has a routine ophthalmic examination, another applanation tonometry, gonioscopy, and peripheral and central visual-field tests. The patient is then given an appointment for tonography and any provocative tests which may be indicated. Finally, the patients are seen by the ophthalmologist and myself in consultation to decide what diagnostic category they belong to, and a report of the findings is sent to the patient's general practitioner so that he can arrange any treatment required, normally by referring the patient to an ophthalmologist.

\section{Results}

Using the above criteria, 98 cases were referred out of the first thousand screened. Eleven failed to attend for assessment and 14 were diagnosed as having definite glaucoma, 3 
of these being closed-angle cases. Thirty-nine showed no abnormality, and the largest single reason for referring in these was a family history of glaucoma (in 15 of them). The remaining cases we have divided into those with raised tension and a coefficient of outflow of less than 0.11 , who we feel are probably cases of early chronic simple glaucoma; cases with a tension on more than one occasion of over 21 $\mathrm{mm} . \mathrm{Hg}$ on the applanation tonometer and in whom the coefficient of outflow is within normal limits (these we have called ocular hypertension); and cases with a tension within normal limits but a low coefficient of outflow.

I am aware that the second and third categories may well contain patients who will develop classical chronic simple glaucoma at a later late, and we intend to make a special study of this group.

One important point, which can only be studied retrospectively after a fairly large number of patients have been surveyed, is the accuracy of the criteria chosen for referring patients from the screening procedure for further investigation. The tables which follow show the assessment findings, analysed according to the reasons for referral (Table I) from the screening clinic.

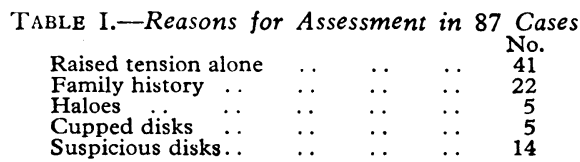

Table II gives the findings in patients with a tension of 21 $\mathrm{mm} . \mathrm{Hg}$ in one eye only : four were normal, but when the tension was over $22 \mathrm{~mm}$. $\mathrm{Hg}$ only one was entirely normal. Similarly, of patients with a tension of $21 \mathrm{~mm}$. $\mathrm{Hg}$ in both eyes (Table III), two were normal, but with a tension of 22 or over only 3 out of 16 were found to be normal. An applanation reading of $21 \mathrm{~mm}$. $\mathrm{Hg}$ is therefore a realistic figure for the upper limit of normal. This does not, of course, exclude the possibility that a few patients with tensions below this level may have glaucoma.

Table IV shows the results of investigations in people with a family history of glaucoma. It is rather surprising to find that only one patient of those with a family history but no other suspicious signs proved to have glaucoma.

TABLE II.-Raised Tension in One Eye (20 Cases) $21 \mathrm{~mm}$. $\mathrm{Hg}$ (11 cases)

Chronic simple glaucoma

Low tension with low outflow

Ocular hypertension

22-25 mm. Hg (8 cases):

Normal

Low tension and lowic simple

Ocular hypertension

25-30 mm. Hg (1 case) :

Unilateral traumatic glaucoma

TaBle III.-Raised Tension in Both Eyes (21 Cases)

$21 \mathrm{~mm}$. $\mathrm{Hg}$ (5 cases) Normal

Ocular hypertension

$\mathrm{mm} . \mathrm{Hg}$ (9 cases)

Normal

Traumatic glaucom

Probable early chronic simple glaucoma

25-30 $\mathrm{mm}$.

Norma

Chronic simple glaucom

Ocular hypertension

Over $30 \mathrm{~mm}$. Hg ( 2 cases)

Probable early chronic simple glaucoma

TABLE IV.-Family History of Glaucoma (22 Cases) Alone (16 cases):

Impending early closed-angle glaucoma $\cdots \quad \ldots$

.

Norma

Impending early closed-angle glaucoma

Probable early chronic simple glaucom Ocular hypertension

Combined with suspicious disk (i case):
Normal
Five patients (Table V) gave a history of haloes, but only in the two cases in which the tension was raised was there any evidence of glaucoma. Patients with disk changes (Table VI) combined with raised tension were all glaucomatous, but of the 11 with suspicious disks alone only two showed abnormality in the form of a poor outflow. These two may represent cases of early chronic simple glaucoma in patients whose blood supply to the disk is affected by a level of tension still within the normal range.

\section{TABLE V.-Haloes (5 Cases) \\ $\begin{array}{lr}\text { Alone }(3 \text { cases }): & \text { No. } \\ \text { Normal ... . . . } 0 .: & 3 \\ \text { Combined with raised tension }(2 \text { cases }): & 2 \\ \text { Chronic closed-angle glaucoma } & 2\end{array}$}

TABLE VI.-Suspicious Disks (14 Cases)

Alone (11 cases):

Normal

Low tension with poor outflow

Combined with raised tension ( 3 cases):

Chronic simple glaucoma Unilateral traumatic glaucoma

Pathologically cupped disks ( 5 cases); all had raised tension:

Chronic simple glaucoma

Chronic closed-angle glaucoma

Table VII shows some of the other interesting ocular conditions which were found in the first thousand cases.

TABLE VII.-Cases of Interest Seen During the Survey Recurrence of rodent ulcer of lower lid Malignant hypertension Heterochromic cyclitis

Chronic anterior uveit

? Early iris neoplasm

mith large multiple staphylomata

neuritis with tabes dorsalis

\section{Discussion}

Reviewing our criteria for assessment in the light of these figures, it is clear that a raised tension is by far the best single criterion for suspecting glaucoma. Pathological cupping of the disk comes second in importance, but half the cases of glaucoma would have been missed if this had been the only criterion. Two of the three patients with closed-angle glaucoma would have been suspected from a history of haloes, but in both of these the tension was raised.

It would seem, therefore, that the most practical single procedure for mass surveys for glaucoma is tonometry. The perfect tonometer does not yet exist, and, even given a highly accurate tonometer, there are still some problems to be solved. It is well known that there is a diurnal variation in intraocular pressure, and our observations at Bedford suggest that the mean levels of pressure are higher in the morning than in the evening. There is also some evidence that the intraocular pressure in women varies throughout the menstrual cycle. Again, an increase with age has been reported. Are we justified, therefore, in accepting any single upper limit of ocular tension without reference to such factors? How many cases of glaucoma have we missed because they were examined when one or a combination of these, and other, factors reduced the tension to $20 \mathrm{~mm}$. $\mathrm{Hg}$ ? I think there is a good case for modifying the criteria for each individual in the light of the probable diurnal variation, age, and, in pre-menopausal women, the stage in the menstrual cycle.

How far have we succeeded in fulfilling the aims of this survey? First, the incidence of glaucoma : in the 2,000 people screened to date 32 cases of definite glaucoma have been detected-an incidence of $1.6 \%$, which agrees well with figures from many surveys which have been done in the United States. We should not, however, at present accept this figure as representing the true incidence of glaucoma in the population. Our volunteers do not necessarily comprise a random sample of the population. It could be argued with some justice that people who are aware that their eyes are 
defective are more likely to volunteer than those who are untroubled by ocular symptoms. Again, in the publicity campaign it was reported that people whose relatives have glaucoma are more likely to be affected themselves, and I feel sure many have volunteered on this account. These factors would tend to exaggerate the true incidence of glaucoma ; but equally there are other factors which might minimize it. We know that the incidence of glaucoma increases with age, and it is possible that fewer elderly people have volunteered because they are incapacitated in some way or even so blind from glaucoma that they have not read of the scheme in the local press. Again, some who are aware of defective vision may be afraid to have their suspicions confirmed.

Ideally we should enlarge the sample to include the whole population above the age of 40 . Certainly we should see many thousands more and make special efforts to screen the elderly and infirm.

Our second aim was to assess the methods of diagnosis and investigation at present available. We have established that applanation tonometry can be done satisfactorily by a trained technician under the prevailing conditions and that it gives an excellent criterion for assessment. The level of $21 \mathrm{~mm}$. $\mathrm{Hg}$ as a satisfactory criterion is supported by the mean values for the normal eyes of $15.2 \mathrm{~mm}$. Hg (S.D. 2.516) for the right eyes and 14.9 (S.D. 2.533) for the left eyes. Two standard deviations above the mean give values of 20.2 and $19.96 \mathrm{~mm}$. $\mathrm{Hg}$ for the right and left eyes respectively.

So far as other diagnostic methods are concerned, some new techniques are being evaluated at the moment.

From the organization point of view we can say that, with adequate publicity and good relations between the population and the local health services, volunteers are forthcoming for screening programmes, and such programmes can be carried out successfully from a public health clinic.

So far as research is concerned, we are collecting an interesting group of patients who have some anomaly of aqueous dynamics but who, up to the present, do not show the classical picture of glaucoma.

Perhaps one of the most important things we have learnt is that the investigation of the borderline group is very timeconsuming and difficult. Our experience suggests that as much time must be spent on investigating the $8 \%$ of patients referred for assessment as is spent in screening the rest. This must be borne in mind in any discussion of glaucomascreening on a national scale. The investigation of $8 \%$ of the whole population over the age of 40 (perhaps some 20 million persons) would mean investigating over one and a half million people. As there are approximately 350 consultant ophthalmologists in the country, and assuming the burden was shared equally, each would have some 4,500 glaucoma suspects to deal with-a sobering thought. It seems clear that if a nation-wide screening programme is evolved special glaucoma clinics must be provided to assess doubtful cases and supervise the results of treatment.

\section{Summary}

The organization, method of screening, criteria for selection of cases for reassessment, and preliminary results of the current glaucoma survey at Bedford are described. Definite glaucoma was diagnosed in $1.6 \%$ of the first 2,000 cases. As much time must be spent on assessment of the $8 \%$ of doubtful cases as on screening the rest.

I should like to acknowledge here that much of the success of this survey has resulted from the smooth organization provided by Dr. C. Sharp, the Medical Officer of Health for Bedford, and his staff, and the high standard maintained by the ophthalmologist, Dr. J. E. Wright, and the technician, Miss E. Verkoczy.

\section{REFERENCE}

Sorsby, A. (1956). Blindness in England, 1951-1954, pp. 6, 13. H.M.S.O., London. (Ministry of Health Report.)

\title{
Long-term Survival with Six Inches of Small Intestine
}

\author{
CHARLOTTE M. ANDERSON,* M.D., M.SC., M.R.A.C.P.
}

\author{
[With Special Plate]
}

Brit. med. F., 1965, 1, 419-422

Much has been written about the fate of patients who have had considerable lengths of small intestine removed surgically, and it seems that survival without severe malabsorption and malnutrition can certainly be expected when up to $50 \%$ has been removed. Pietz (1956) offers this opinion from an appraisal of the literature to that date, but states that fair health can be maintained if only $2 \mathrm{ft}$. $(60 \mathrm{~cm}$.) of intestine remains. However, long-term survival when less than $2 \mathrm{ft}$. $(60 \mathrm{~cm}$.) of small intestine has been left behind is very uncommon (Levin, Zamcheck, and Gottlieb, 1961). The purpose of this paper is to record the survival in reasonably good health and nutrition of a girl, now 22 years of age, who had all but

\footnotetext{
* Head of Gastroenterological Research Unit, Royal Children's Hospital
} Research Foundation, Melbourne, Australia. approximately 6 in. $(15 \mathrm{~cm}$.) of her small intestine removed seven and a half years ago.

\section{Case Report}

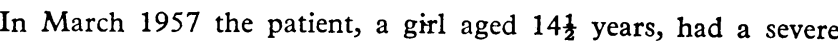
attack of abdominal pain associated with vomiting. She had suffered from mild attacks of a similar nature since the age of 10 , but otherwise had been well and had grown normally. The pain and vomiting continued for some days and she became very ill, being admitted to hospital in a state of dehydration and circulatory failure. General peritonitis was diagnosed and after resuscitation an emergency laparotomy was performed. Volvulus of the small intestine had occurred and the whole of the bowel concerned in the volvulus was gangrenous. All but 3 in. $(7.5 \mathrm{~cm}$.) of the jejunum beyond the duodeno-jejunal flexture, and 3 in. $(7.5 \mathrm{~cm}$.) of ileum proximal 